\title{
A New Approach to the Management of Anemia in CKD Patients: A Review on Roxadustat
}

Kimberly Becker · Maha Saad

Received: January 16, 2017 / Published online: March 13, 2017

(C) The Author(s) 2017. This article is published with open access at Springerlink.com

\begin{abstract}
This article informs the reader of the current information available on a novel therapeutic agent and new class of drug for the treatment of anemia. The data show promising results for alternative erythropoietin-stimulating agents and offers a time line of when Phase III data will be available. The information on this new drug and new drug class will change how nephrologists approach treating anemia within their patients.
\end{abstract}

Keywords: Anemia; Chronic kidney disease; Erythropoietin-stimulating agent; Dialysis; Hematology; Hemoglobin; HIF-Inhibitor; Non-dialysis; Nephrology; Roxadustat

\section{INTRODUCTION}

An important function of the kidneys is the production of erythropoietin, a hormone that stimulates the production of red blood cells. In disease states such as chronic kidney disease

Enhanced content To view enhanced content for this article go to http://www.medengine.com/Redeem/ 54F7F0600AD0E5F3.

K. Becker $(\bowtie) \cdot$ M. Saad

St. John's University, Jamaica, NY, USA

e-mail: kimberly.anne.becker@gmail.com
(CKD), the kidneys are not functioning at capacity and there can be a disruption in the production of erythropoietin that can lead to anemia. Other causes of anemia in CKD are iron deficiency and inflammation [1, 2]. Anemia may be present in all stages of CKD and increases in prevalence as CKD progresses [2,3]. Current treatment of anemia includes iron replacement and use of erythropoeitin (EPO) analogs. The FDA has issued warnings on the use of EPO analogs due to a greater risk of death and serious cardiovascular events [4].

Roxadustat, also known as FD-4592, is a first-in-class small molecule oral hypoxia-inducible factor prolyl hydroxylase inhibitor (HIF-PHI) currently in Phase III development [4, 5]. Fibrogen, Inc. has collaborated with Astellas Pharma Inc. and AstraZeneca in the development and commercialization of roxadustat and estimates completion of the Phase III trials for U.S approval in 2017 [6]. This article is based on previously conducted studies and does not involve any new studies of human or animal subjects performed by the authors.

\section{Scientific Summary}

\section{Pharmacodynamics}

Roxadustat offers a novel way of utilizing the body's natural compensatory mechanisms in response to hypoxia $[3,4,7]$. Hypoxia-inducible factors (HIF) are transcription factors that 
regulate expression of genes that stimulate erythropoiesis. When the body has normal oxygen levels, HIF-specific prolyl hydroxylase (HIF-PH) will degrade HIF $\alpha$. In hypoxic conditions, such as at increasing altitudes, HIF-PH activity is decreased to compensate for the reduced oxygen concentration. Roxadustat is a HIF-PH Inhibitor (HIF-PHI) that inhibits the degradation of HIF $\alpha$. When HIF $\alpha$ accumulates, it dimerizes with HIF $\beta$ and translocates to the nucleus to activate the transcriptional response to hypoxia by promoting erythropoiesis. Phase I and phase II roduxadustat trials have provided evidence that erythropoiesis was stimulated by an accumulation of plasma endogenous erythropoietin and suppression of hepcidin, an indirect regulator of iron absorption and utilization [4]. When hepcidin levels are suppressed, intestinal absorption of iron increases and the release of iron from storage sites are utilized.

\section{Pharmacokinetics}

Roxadustat is orally bioavailable with an estimated half-life of $12 \mathrm{~h}$ [7]. In a placebo-controlled dose-ranging study (NCT00761657), selected patients who were being studied for treatment efficacy of roxadustat in NDD-CKD were also evaluated for pharmacokinetic and pharmacodynamics parameters [4]. Evaluations included measurement of endogenous erythropoietin (eEPO), iron indices including total iron-binding capacity (TIBC) and transferrin saturation (TSAT), as well as hepcidin levels. When roxadustat was administered in doses of either 1.0 or $2.0 \mathrm{mg} / \mathrm{kg}$ twice weekly (BIW) or three times weekly (TIW), eEPO started to increase within $4 \mathrm{~h}$ of the dose and peaked at $10 \mathrm{~h}$ post-dose. Endogenous EPO fell back to baseline at 24-48 h post-dose regardless of the dose given. Peak EPO increased with dose but not with frequency. At 6-week follow-up, there was a decrease in TSAT and an increase in TIBC in the roxadustat group, signifying increased iron utilization, as well as a significant decrease in hepcidin levels.

\section{Therapeutic Trials}

Anemia Treatment in Nondialysis-Dependent $C K D$ A Phase II randomized placebo-controlled dose-ranging and pharmacodynamics study of roxadustat (NCT00761657) evaluated the treatment of anemia in patients with CKD Stages 3-4 (eGFR: $15-59 \mathrm{~mL} / \mathrm{min} / 1.73 \mathrm{~m}^{2}$ ) with anemia $(\mathrm{Hb} \leq 11.0 \mathrm{~g} / \mathrm{dL})$ [4]. Patients were enrolled to receive doses of either $0.7,1.0,1.5$, or $2.0 \mathrm{mg} / \mathrm{kg}$ and were then randomized in a $3: 1$ ratio to receive roxadustat or placebo administered as either BIW for 4 weeks or TIW for 26 days. Patients were followed for up to 12 weeks post-treatment. Study patients were not permitted to receive EPO analogous, intravenous iron, androgens, or RBC transfusion. If patients were on oral iron, they were kept at the original dose; oral iron was also given at the investigators' discretion. The study defined $\mathrm{Hb}$ response as an increase in $\mathrm{Hb} \geq 1 \mathrm{~g} / \mathrm{dL}$ at any time from Day 1 to the 2-week follow-up. Mean change in $\mathrm{Hb}$ was measured at the end of week 6. A total of 117 patients were randomized and had comparable baseline characteristics with the exception of some differences between sex and race between groups. Hb response was higher in patients who received roxadustat compared to placebo. In pooled placebo groups, $13 \%$ of patients had a $\mathrm{Hb}$ response compared to roxadustat given at $0.7 \mathrm{mg} / \mathrm{kg}$ BIW (60\%), $0.7 \mathrm{mg} / \mathrm{kg}$ TIW (40\%), $1.0 \mathrm{mg} / \mathrm{kg}$ BIW (80\%), $1.0 \mathrm{mg} / \mathrm{kg}$ TIW (91\%), and 100\% response rates in patients who received doses of 1.5 and $2.0 \mathrm{mg} / \mathrm{kg}$ administered as either BIW or TIW. The rates of $\mathrm{Hb}$ response were dependent on the dose administered. When patients received $1.5-2.0 \mathrm{mg} / \mathrm{kg}$ TIW, they achieved a faster response in $\mathrm{Hb}$ compared to BIW groups. A total of 52 adverse events were reported in pooled placebo and roxadustat groups. These AEs were mostly related to the disease state and did not have any clinical differences between the two groups. Serious AEs (SAEs) were reported by 5\% of patients who received roxadustat and $4 \%$ of patients who received placebo. SAEs included vascular access complications, femoral neck fracture, noncardiac chest pain, and dyspnea. There were no incidences of cardiovascular events, seizure, or thromboembolism reported during the study. There were two recorded episodes of moderate exacerbation of hypertension, excessive fluid weight, and one episode of elevated ALT, AST, and bilirubin that were deemed unrelated to the study drug. The most common side effects of roxadustat were 
diarrhea, headache, back pain, and fatigue. This evidence is limited to the small population size studied and the short treatment duration.

\section{Anemia Treatment in Dialysis-Dependent} $C K D$ An open label, randomized study evaluated the effect of roxadustat therapy on anemia in dialysis patients and the exogenous iron requirements [3]. A total of 60 patients were enrolled: 24 patients undergoing hemodialysis (HD) received no exogenous iron supplement, 12 HD patients and 12 peritoneal dialysis patients received oral iron, and $12 \mathrm{HD}$ patients given intravenous (IV) iron. The average oral iron dose was $71 \pm 50 \mathrm{mg}$, while the IV iron dose was 50 or $62.5 \mathrm{mg}$ weekly. The mean weekly dose, administered three times a week, of roxadustat $(4-4.3 \mathrm{mg} / \mathrm{kg}$ ) varied, but did not significantly differ amongst groups and was adjusted based on hemoglobin $(\mathrm{Hb})$ response. The patients included in this study had an average age of $50 \pm 15$ years, were $52 \%$ men, the majority (90\%) white, with an average time since first dialysis of $2.2 \pm 0.9$ months and a baseline $\mathrm{Hb}$ of $8.3 \pm 1 \mathrm{~g} / \mathrm{dL}$. Mean $\mathrm{Hb}$ increases of $\geq 2 \mathrm{~g} / \mathrm{dL}$ within 7 weeks was achieved in each group. In addition, the overall mean change from baseline of $\mathrm{Hb}$ was $3.1 \pm 0.2 \mathrm{~g} / \mathrm{dL}$. The $\mathrm{Hb}$ response was greater in patients receiving iron supplement than those who did not; however, the response was similar regardless of the iron route of administration. Hepcidin levels decreased in all iron regimen groups, with the largest drop (80\%) observed in patients on HD not receiving iron. Other parameters, such as transferring saturation (TSAT) levels and reticulocyte $\mathrm{Hb}$ content, did not change in the groups receiving iron; however, a decrease was observed in patients not receiving iron. Adverse events (AEs) were reported in 50\% of all patients, and these AEs were typical of the patient population undergoing dialysis. Most common AEs included hypertension (10\%) and decreased TSAT (6.7\%); all other AEs had an incidence of less than 5\%. Two patients had abnormal electrogardiograms at baseline, while, through the treatment, one patient developed a first-degree arteriovenous block, and two patients had a transient elevation in liver function tests unrelated to roxadustat. Two deaths were reported and determined to be unrelated to roxadustat. This study has a number of limitations, including small sample size, open label design, short treatment durations and lack of comparison with standard of care such as erythropoetin analogue and iron therapy.

Anemia Treatment in Patients with Dialysis-Dependent CKD who Previously had $\mathrm{Hb}$ Levels Maintained with Epoetin Alfa A phase II, randomized, open-label, dose-ranging, active control study (NCT01147666) evaluated the safety and efficacy of roxadustat in maintaining $\mathrm{Hb}$ levels in patients on maintenance dialysis therapy, and who had maintained Hb levels with the IV EPO analog, epoetin alfa [7]. The study also evaluated the optimum starting dose and dose adjustment regimens of roxadustat in this patient population. ESRD adult patients were included in the study if they were maintained on a three times weekly dialysis schedule for a duration of $\geq 4$ months with Hb 9.0-13.5 g/dL for 8 weeks, and stable EPO analogs at dosages $\leq 450 \mathrm{U} / \mathrm{kg} /$ weekk for 4 weeks. The study defined a $\mathrm{Hb}$ response rate as patients whose $\mathrm{Hb}$ did not decrease $>0.5 \mathrm{~g} / \mathrm{dL}$. Part I of the study was conducted in 52 patients over 6 weeks with an 8 -week follow-up to evaluate the roxadustat doses of $1.0,1.5,1.8$, or $2.0 \mathrm{mg} / \mathrm{kg}$ three times a week compared to continuation of EPO. Part 2 was 19 weeks with a follow-up of 4 weeks which evaluated 90 patients in 6 cohorts to evaluate roxadustat with various starting doses compared to continuation of EPO analog (median dose of $\mathrm{EPO}$ analog $=90 \mathrm{U} / \mathrm{kg} /$ week). In part I of the study, patients who received the lowest dose of roxadustat $1.0 \mathrm{mg} / \mathrm{kg}$ TIW had a comparable $\mathrm{Hb}$ response rate of $-0.5 \mathrm{~g} / \mathrm{dL}$ or greater from baseline compared to EPO analog. Roxadustat doses $\geq 1.5 \mathrm{mg} / \mathrm{kg}$ TIW maintained a $\mathrm{Hb}$ response rate of $79 \%$ compared to a $33 \%$ response rate in patients who received EPO ana$\log (p=0.03)$. Pooled roxadustat patients had an overall mean increase in $\mathrm{Hb}$ from baseline of $0.3 \mathrm{~g} / \mathrm{dL}$ compared to a decrease of $1.0 \mathrm{~g} / \mathrm{dL}$ in patients who received EPO analog $(p=0.04)$

In Part II of the trial, patients who received any dose of roxadustat achieved a maintenance $\mathrm{Hb} \geq 11 \mathrm{~g} / \mathrm{dL}$ over the last 4 weeks of the 
19-week treatment compared to EPO analog (51\% and $36 \%$, respectively). Individual roxadustat dose cohorts treated for 19 weeks did not significantly differ from EPO analog treated patients in change in $\mathrm{Hb}$ at any time. $\mathrm{Hb}$ was maintained with a mean roxadustat dose of $1.68 \pm 0.65 \mathrm{mg} / \mathrm{kd}$ TIW with starting doses based on weight or weight tiers. C-reactive protein (CRP), which increases during an inflammatory state, was correlated with increased EPO analog dose used, but there were no differences in dose requirements of roxadustat in patients with inflammation. There was a greater increase in measured blood EPO levels $12 \mathrm{~h}$ post-treatment in patients who received $\mathrm{EPO}$ analog compared to a modest $\mathrm{EPO}$ increase in patients who received roxadustat. Pooled patients who received roxadustat had decreases in hepcidin as well as increases in reticulocyte $\mathrm{Hb}$ content. There were no significant differences between the two groups in regards to change in TSAT, serum iron, and total cholesterol. Safety profiles were comparable between roxadustat and EPO analog. In the safety population, $24.1 \%$ and $17 \%$ of patients experienced at least $1 \mathrm{SAE}$ with roxadustat and EPO analog, respectively. Of roxadustat patients, $63.9 \%$ of patients experienced at least $1 \mathrm{AE}$ and $12 \%$ experienced a cardiovascular composite safety endpoint compared to patients who received EPO analog, 61.1\% and $17 \%$, respectively.

Roxadustat was effective at maintaining $\mathrm{Hb}$ levels in patients with ESRD compared to a wide range of EPO analog doses not receiving IV iron. Roxadustat was more effective at doses $\geq 1.5 \mathrm{mg} / \mathrm{kg}$ TIW compared to lower and less frequent doses of roxadustat. Roxadustat and EPO analog had comparable safety profiles.

\section{Adverse Events}

Roxadustat was well tolerated in Phase II trials, and most documented adverse events were typical for patients with CKD [3, 4]. Common documented side effects were diarrhea, headache, back pain, fatigue, and increases in blood pressure and liver enzymes $[3,4,7]$. Cardiovascular events were comparable in patients who received roxadustat compared to EPO analog; however, larger trials must be conducted to confirm these results [7]. Since HIF-inhibitors effectively increase $\mathrm{EPO}$ and $\mathrm{Hb}$, it can be hypothesized that there may be potential long-term cardiovascular and/or malignant side effects that may become apparent with longer duration trials and post-marketing analysis.

\section{Ongoing Clinical Trials}

There are currently 9 phase III clinical trials studying the safety, efficacy, and long-term effects of roxadustat in patients with various degrees of $\mathrm{CKD}$; including dialysis, non-dialysis-dependent, and on newly initiated dialysis [6] (Table 1). The trials are comparing the use of roxadustat in comparison to either placebo or an active control such as darbepoetin alfa and epoetin alfa. The long-term effects of roxadustat are currently being studied in an open-label 5-year extension study to evaluate the safety and efficacy of roxadustat for longterm maintenance use. This study should yield some insight into the potential cardiovascular and malignant side effects that may occur.

\section{CONCLUSIONS}

Phase II roxadustat trials have demonstrated the effectiveness of roxadustat in the treatment of anemia (defined as $\mathrm{Hb}$ increase $\geq 1 \mathrm{~g} / \mathrm{dL}$ ) and maintenance of $\mathrm{Hb}$ levels (defined as $\mathrm{Hb}$ not falling more than $0.5 \mathrm{~g} / \mathrm{dL}$ ) in patients with ND-CKD and dialysis-dependent CKD [3, 4, 7]. Roxadustat has been documented to increase endogenous EPO and suppress hepcidin which may have positive effects on the utilization and mobilization of iron $[3,4]$. The efficacy of roxadustat is dose-dependent. Doses higher than $1.5 \mathrm{mg} / \mathrm{kd}$ TIW may possibly be an alternative to EPO analog; however, since the use of IV iron was prohibited during the study, the ESA may not have been able to raise $\mathrm{Hb}$ by its full potential [7]. The safety profile of roxadustat is comparable to that of EPO analog; however, data from larger studies will have to confirm these results. Common adverse events experienced with the roxadustat include diarrhea, headache, back pain, and fatigue [4]. The data presented are limited because of the small sample sizes and short durations of the Phase II trials reviewed [2, 3, 7]. 
Table 1 Ongoing phase III Roxadustat clinical trials

\begin{tabular}{|c|c|c|c|}
\hline Trial Name & Comparator(s) & $\begin{array}{l}\text { ClinicalTrials.gov } \\
\text { identifier }\end{array}$ & $\begin{array}{l}\text { Estimated } \\
\text { primary } \\
\text { completion date }\end{array}$ \\
\hline $\begin{array}{l}\text { Roxadustat in the treatment of anemia in chronic kidney } \\
\text { disease (CKD) patients, not on dialysis, in comparison to } \\
\text { darbepoetin alfa (dolomites) }\end{array}$ & $\begin{array}{l}\text { Darbepoetin } \\
\text { Alfa }\end{array}$ & NCT02021318 & July 2017 \\
\hline $\begin{array}{l}\text { Evaluation of efficacy and safety of Roxadustat in the } \\
\text { treatment of anemia in stable dialysis subjects }\end{array}$ & Epoetin Alfa & NCT02273726 & June 2017 \\
\hline $\begin{array}{l}\text { Safety and efficacy study of Roxadustat to treat anemia in } \\
\text { patients with chronic kidney disease, on dialysis }\end{array}$ & Epoetin Alfa & NCT02174731 & February 2017 \\
\hline $\begin{array}{l}\text { Roxadustat in the treatment of anemia in chronic kidney } \\
\text { disease patients not requiring dialysis (ALPS) }\end{array}$ & Placebo & NCT01887600 & January 2018 \\
\hline $\begin{array}{l}\text { Safety and efficacy study of Roxadustat to treat anemia in } \\
\text { patients with chronic kidney disease (CKD), not on dialysis }\end{array}$ & Placebo & NCT02174627 & March 2017 \\
\hline $\begin{array}{l}\text { Roxadustat in the treatment of anemia in end stage renal } \\
\text { disease (ESRD) patients on stable dialysis (pyrenees) }\end{array}$ & $\begin{array}{l}\text { Darbepoetin } \\
\text { Alfa } \\
\text { Epoetin Alfa }\end{array}$ & NCT02278341 & June 2017 \\
\hline $\begin{array}{l}\text { Safety and efficacy study for treatment of anemia in ESRD } \\
\text { newly initiated dialysis patients (himalayas) }\end{array}$ & Epoetin Alfa & NCT02052310 & June 2017 \\
\hline $\begin{array}{l}\text { A study of FG- } 4592 \text { for the treatment of anemia in chronic } \\
\text { kidney disease patients not receiving dialysis }\end{array}$ & Placebo & NCT01750190 & June 2017 \\
\hline $\begin{array}{l}\text { Open label extension study for the long-term efficacy and } \\
\text { safety of FG- } 4592 \text { in dialysis and non-dialysis chronic } \\
\text { kidney disease patients }\end{array}$ & $\mathrm{N} / \mathrm{A}$ & NCT01630889 & October 2018 \\
\hline
\end{tabular}

There are multiple small molecule HIF-inhibitors currently in clinical development [8]. Drugs in this class share the same mechanism of action but differ in dosing and frequency. If the phase III trials reveal positive results in 2017, the manufacture of roxadustat expects to file an NDA in 2018 [5, 6]. This will make roxadustat a first-in-class HIF-inhibitor.

\section{ACKNOWLEDGEMENTS}

No funding or sponsorship was received for this study or publication of this article. All named authors meet the International Committee of Medical Journal Editors (ICMJE) criteria for authorship for the manuscript, take responsibility for the integrity of the work as a whole, and have given final approval for the version to be published.

Disclosures. Kimberly Becker and Maha Saad have nothing to disclose.

Compliance with Ethics Guidelines. This article is based on previously conducted studies and does not involve any new studies of human or animal subjects performed by the authors.

Data Availability. Data sharing is not applicable to this article as no datasets were generated or analyzed during the current study. 
Open Access. This article is distributed under the terms of the Creative Commons Attribution-NonCommercial 4.0 International License (http://creativecommons.org/licenses/ by-nc/4.0/), which permits any noncommercial use, distribution, and reproduction in any medium, provided you give appropriate credit to the original author(s) and the source, provide a link to the Creative Commons license, and indicate if changes were made.

\section{REFERENCES}

1. Onken J., Bregman B., Harrington RA., et al. Ferric carboxymaltose in patients with iron deficiency anemia and impaired renal function: the REPAIR-IDA trial. Nephrol Dial Transplant. 2013.

2. Stauffer ME, Fan T. Prevalence of anemia in chronic kidney disease in the United States. PLoS ONE. 2014;9(1):e84943.

3. Besarab A, Chernyavskaya E, Motylev I, et al. Roxadustat (FG-4592): correction of anemia in incident dialysis patients. J Am Soc Nephrol. 2016;27(4):1225-33.

4. Besarab A, Provenzano R, Hertel J, et al. Randomized placebo-controlled dose-ranging and pharmacodynamics study of roxadustat (FG-4592) to treat anemia in nondialysis-dependent chronic kidney disease (NDD-CKD) patients. Nephrol Dial Transplant. 2015;30(10):1665-73.

5. FibroGen, Inc. Home-FribroGen, Inc. http://www. fibrogen.com/. Accessed 18 Nov 2016.

6. U.S. National Institutes of Health. Roxadustat. ClinicalTrials.gov. http://www.clinicaltrials.gov. Accessed 18 Nov 2016.

7. Provenzano R, Besarab A, Wright S, et al. Roxadustat (FG-4592) versus epoetin alfa for anemia in patients receiving maintenance hemodialysis: a Phase 2 , randomized, 6- to 19-week, open-label, active-comparator, dose-ranging, safety and exploratory efficacy study. Am J Kidney Dis. 2016;67(6):912-24.

8. U.S. National Institutes of Health. HIF inhibitor. ClinicalTrials.gov. http://www.clinicaltrials.gov. Accessed 15 Feb 2017. 